\title{
New Precise Measurement of the Pion Weak Form Factors in $\pi^{+} \rightarrow e^{+} \nu \gamma$ Decay
}

\author{
M. Bychkov, ${ }^{1, *}$ D. Počanić, ${ }^{1, \dagger}$ B. A. VanDevender, ${ }^{1}$ V. A. Baranov, ${ }^{2}$ W. Bertl,${ }^{3}$ Yu. M. Bystritsky, ${ }^{2}$ E. Frlež, ${ }^{1}$ \\ V. A. Kalinnikov, ${ }^{2}$ N. V. Khomutov, ${ }^{2}$ A. S. Korenchenko, ${ }^{2}$ S. M. Korenchenko, ${ }^{2}$ M. Korolija, ${ }^{4}$ T. Kozlowski, ${ }^{5}$ \\ N. P. Kravchuk, ${ }^{2}$ N. A. Kuchinsky, ${ }^{2}$ W. Li, ${ }^{1}$ D. Mekterović, ${ }^{4}$ D. Mzhavia, ${ }^{2,6}$ S. Ritt, ${ }^{3}$ P. Robmann, ${ }^{7}$ \\ O. A. Rondon-Aramayo, ${ }^{1}$ A. M. Rozhdestvensky, ${ }^{2}$ T. Sakhelashvili, ${ }^{3}$ S. Scheu, ${ }^{7}$ U. Straumann, ${ }^{7}$ I. Supek, ${ }^{4}$ \\ Z. Tsamalaidze, ${ }^{2,6}$ A. van der Schaaf, ${ }^{7}$ E. P. Velicheva, ${ }^{2}$ V. P. Volnykh, ${ }^{2}$ Y. Wang, ${ }^{1}$ and H.-P. Wirtz ${ }^{3}$ \\ ${ }^{1}$ Department of Physics, University of Virginia, Charlottesville, Virginia 22904-4714, USA \\ ${ }^{2}$ Joint Institute for Nuclear Research, RU-141980 Dubna, Russia \\ ${ }^{3}$ Paul Scherrer Institut, Villigen PSI, CH-5232, Switzerland \\ ${ }^{4}$ Institut "Rudjer Bošković," HR-10000 Zagreb, Croatia \\ ${ }^{5}$ Instytut Problemów Jadrowych im. Andrzeja Soltana, PL-05-400 Świerk, Poland \\ ${ }^{6}$ Institute for High Energy Physics, Tbilisi State University, GUS-380086 Tbilisi, Georgia \\ ${ }^{7}$ Physik-Institut, Universität Zürich, CH-8057 Zürich, Switzerland
}

(Received 15 April 2008; revised manuscript received 10 June 2009; published 30 July 2009)

\begin{abstract}
We have measured the $\pi^{+} \rightarrow e^{+} \nu \gamma$ branching ratio over a wide region of phase space, based on a total of 65460 events acquired using the PIBETA detector. Minimum- $\chi^{2}$ fits to the measured $\left(E_{e^{+}}, E_{\gamma}\right)$ energy distributions result in the weak form factor value of $F_{A}=0.0119(1)$ with a fixed value of $F_{V}=0.0259$. An unconstrained fit yields $F_{V}=0.0258(17)$ and $F_{A}=0.0117(17)$. In addition, we have measured $a=$ $0.10(6)$ for the dependence of $F_{V}$ on $q^{2}$, the $e^{+} \nu$ pair invariant mass squared, parametrized as $F_{V}\left(q^{2}\right)=$ $F_{V}(0)\left(1+a q^{2}\right)$. The branching ratio for the kinematic region $E_{\gamma}>10 \mathrm{MeV}$ and $\theta_{e^{+} \gamma}>40^{\circ}$ is measured to be $B^{\text {expt }}=73.86(54) \times 10^{-8}$. Earlier deviations we reported in the high- $E_{\gamma}-l o w-E_{e^{+}}$kinematic region are resolved without a tensor term. We also derive new values for the pion polarizability $\alpha_{E}=2.78(10) \times$ $10^{-4} \mathrm{fm}^{3}$ and neutral pion lifetime $\tau_{\pi 0}=(8.5 \pm 1.1) \times 10^{-17} \mathrm{~s}$.

DOI: 10.1103/PhysRevLett.103.051802

PACS numbers: 13.20.Cz, 11.30.Rd, 14.40.Aq
\end{abstract}

Radiative pion decay $\pi^{+} \rightarrow e^{+} \nu \gamma$ (also denoted $\pi_{e 2 \gamma}$ ), where $\gamma$ is a real photon, offers the best means to study pion form factors at zero momentum transfer. Pion form factors provide critical input to low-energy effective theories of the strong interaction, such as chiral perturbation theory (ChPT). Previous experimental studies of the $\pi_{e 2 \gamma}$ decay were constrained by relatively low event statistics and limited kinematic coverage, leaving room for speculative interpretations including an anomalously large tensor interaction term. In this Letter, we report precise new results of a combined analysis of data recorded with the PIBETA detector [1], removing the limitations and ambiguities found in previous work.

In the standard description, the $\pi_{e 2 \gamma}$ decay amplitude consists of the inner bremsstrahlung, structure-dependent $\left(\mathrm{SD}^{ \pm}\right)$, and interference terms. The SD terms are parametrized by vector $\left(F_{V}\right)$ and axial vector $\left(F_{A}\right)$ form factors (see Ref. [2] for a review). The conserved vector current (CVC) hypothesis relates $F_{V}$ to the $\pi^{0}$ lifetime [3-5], yielding $F_{V}=0.0259(9)$ [6]. ChPT calculations [7-10] give a value for $F_{A}$ in the range $0.010-0.012$.

Because of the above noted limitations, authors of previous experimental studies fixed $F_{V}$ at the CVC-predicted value and evaluated $F_{A}$, with resulting relative uncertainties ranging from $\pm 12 \%$ to $\pm 56 \%$ [11-16]. None of these measurements was sensitive to the form factor dependence on the $e^{+} \nu$ pair invariant mass. Because of inconsistencies in the available data, a relatively large non- $(V-A)$ tensor contribution to the SD terms was considered (see Ref. [17] and references therein). Our first measurement [18] reported a significant improvement in the accuracy of $F_{A}$ but also noted a substantial deficit of observed events in the high- $E_{\gamma}-$ low- $E_{e^{+}}$kinematic region, still leaving room for non- $(V-A)$ admixtures.

We performed measurements at the $\pi E 1$ beam line at the Paul Scherrer Institute (PSI), Switzerland, using a stopped $\pi^{+}$beam and the PIBETA large-acceptance CsI calorimeter with central tracking [1]. A total of over $2.0 \times$ $10^{13} \pi^{+}$'s stopped in the active target during the two measuring periods were used in the analysis. The first data set (1999-2001) was collected during the $\pi^{+} \rightarrow$ $\pi^{0} e^{+} \nu\left(\pi_{\beta}\right)$ branching ratio measurement [19] with $\sim 8 \times$ $10^{5}$ pion stops per second. A second sample was recorded in 2004 with an 8 times lower stopping rate. Details of the detector design, performance, and our experimental methods are presented in Refs. [1,18-20].

The PIBETA detector measured shower energies and directions of the positron and photon, thus overdetermining the kinematics of the final three-body state. Key for the $\pi_{e 2 \gamma}$ measurement were the one-arm and two-arm highthreshold triggers $1 \mathrm{HT}$ and $2 \mathrm{HT}$, respectively, requiring that at least one (1HT) or two (2HT) showers register above an effective high threshold of $\sim 54 \mathrm{MeV}$ deposited in the calorimeter, thus reducing a copious $e^{+}$background from the $\pi$ - $\mu$-e decay chain with a continuous energy spectrum ending at $\sim 52.8 \mathrm{MeV}$. In the 1999-2001 data set, the 1HT 
trigger was prescaled 16- or 8-fold. Our $\pi_{e 2 \gamma}$ data set covers three nonoverlapping kinematic regions given in Table I; region I data were acquired with the $2 \mathrm{HT}$ trigger, while the 1HT trigger mapped out regions II and III. In all three regions, the $\pi_{e 2 \gamma}$ decay is strongly dominated by the $\mathrm{SD}^{+}$term proportional to $\left(F_{V}+F_{A}\right)^{2}$ [2]. Candidate $\pi_{e 2 \gamma}$ events exhibit one neutral shower in the calorimeter coincident in time with a positron track. For the events with more than one available combination, i.e., more than one neutral shower or positron track, the pair most nearly coincident in time was chosen. Events incompatible with the $\pi_{e 2 \gamma}$ kinematics were rejected in the final data sample.

Because of the nature of the detector resolution function, the experimental kinematic region contains a fraction of events shifted from a broader kinematic range. We therefore used the reconstructed events in regions I, II, and III to evaluate the branching ratios $B_{\pi e 2 \gamma}^{\text {exp }}$ for the larger regions $A$, $B$, and $C$, respectively, and $O$, the combination of all three regions, by means of Monte Carlo (MC) extrapolation. On the other hand, we calculated the theoretical branching fractions $B_{\pi e 2 \gamma}^{\text {theor }}$ for the same regions numerically using only the input parameters $\left(F_{V}, F_{A}\right.$, and $\left.a\right)$, as described below, and the standard description of the decay, including radiative corrections [21].

Accidental background was dominated by positrons from $\pi_{e 2}$ and $\pi$ - $\mu$-e decays accompanied by an unrelated neutral shower. It was corrected for by subtracting histograms of observables projected using the out-of-time cut $\Delta t_{\text {out }}:\left(5 \mathrm{~ns}<\left|t_{e^{+}}-t_{\gamma}\right|<10 \mathrm{~ns}\right)$ from the in-time histograms projected via the cut $\Delta t_{\text {in }}:\left(\left|t_{e^{+}}-t_{\gamma}\right| \leq 5 \mathrm{~ns}\right)$. The 2004 data sample with lower stopped beam intensity provided an improvement of the signal to accidentalbackground ratios from $1.7: 1$ to $10: 1$ in region II and from 4.4:1 to $30: 1$ in region III.

Non-accidental-background sources were predominately $\pi_{\beta}$ events for which one $\pi^{0}$ decay photon converts in the target, producing a charged track in the detector. Having measured the net yield of the $\pi_{\beta}$ decay events in our detector, we have used a MC simulation to calculate the fraction of $\pi_{\beta}$ decay events misidentified as $\pi_{e 2 \gamma}$. This class of background events contributes $\sim 6.0 \%$ of the signal in the kinematic region I and negligible amounts in

TABLE I. Total energy ranges for kinematic regions used in measurement (I-III, in measured $\mathrm{MeV}_{\text {expt }}$ ) and branching ratio (BR) evaluation $(A-O$, in physical $\mathrm{MeV})$. For regions $B, C$, and $O$, the $e^{+} \gamma$ relative angle is restricted to $\theta_{e^{+} \gamma}>40^{\circ}$; this condition is automatically satisfied for regions I-III and $A$.

\begin{tabular}{cccccc}
\hline \hline & Measurement & \multicolumn{3}{c}{ BR evaluation } \\
Reg. & $E_{e+}^{\text {expt }}$ & $E_{\gamma}^{\text {expt }}$ & Reg. & $E_{e+}$ & $E_{\gamma}$ \\
\hline I & $>51.7$ & $>51.7$ & $A$ & $>50$ & $>50$ \\
II & $20-51.7$ & $>55.6$ & $B$ & $>10$ & $>50$ \\
III & $>55.6$ & $20-51.7$ & $C$ & $>50$ & $>10$ \\
& & $O$ & $>m_{e}$ & $>10$ \\
\hline \hline
\end{tabular}

regions II and III. The $\pi_{\beta}$ contamination was subtracted in the calculation of $\pi_{e 2 \gamma}$ yields.

In order to evaluate the branching ratio of the $\pi_{e 2 \gamma}$ decay in any given kinematic region, for normalization we used the $\pi_{e 2}$ events, recorded in parallel via the one-arm HT calorimeter trigger. This procedure assures the cancellation of the imprecisely known total number of stopped $\pi^{+}$'s, and $\epsilon_{\text {trac }}^{e}$, the combined tracking efficiency of $e^{+}$'s. No statistically significant $e^{+}$energy dependence was observed for $\epsilon_{\text {trac }}^{e}$ [22]. Thus, the experimental branching ratio for the $\pi^{+} \rightarrow e^{+} \nu \gamma$ decay can be calculated from the expression

$$
B_{\pi e 2 \gamma}^{\text {expt }}=B_{\pi e 2} \frac{N_{\pi e 2 \gamma}}{N_{\pi e 2} p_{\pi e 2}} \frac{A_{\pi e 2}}{A_{\pi e 2 \gamma}},
$$

where $N_{\pi e 2 \gamma}\left(N_{\pi e 2}\right)$ is the number of the detected $\pi_{e 2 \gamma}$ $\left(\pi_{e 2}\right)$ events, $A_{\pi e 2 \gamma}\left(A_{\pi e 2}\right)$ is the experimental acceptance for the given decay type incorporating the appropriate cuts, and $B_{\pi e 2}=1.2352(1) \times 10^{-4}$ is the theoretical $\pi_{e 2}$ branching ratio $[23,24]$. The factor $p_{\pi e 2}$ denotes the prescaling of the one-arm HT trigger; it applied to the 19992001 data set only.

The experimental acceptances were calculated in a GEANT3-based MC program [25]. This simulation incorporates a precise description of the PIBETA detector geometry as well as the electronics response of the experimental setup. Figure 1 demonstrates the match between the MC
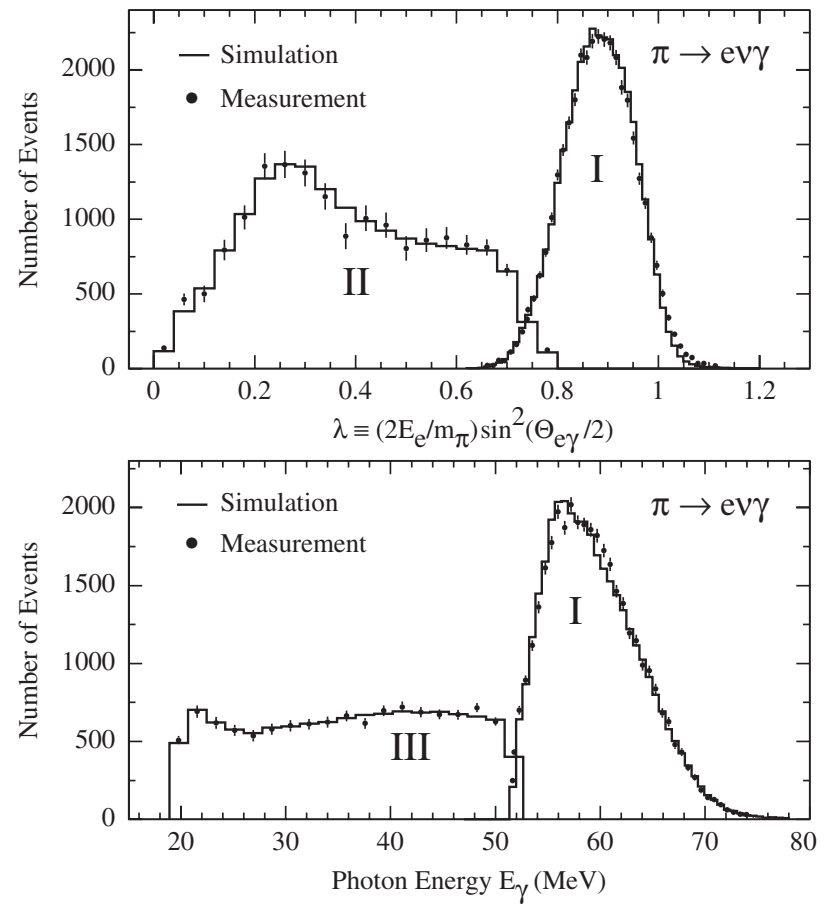

FIG. 1. Data points: Background-subtracted $\pi^{+} \rightarrow e^{+} \nu \gamma$ distribution of the kinematic variable $\lambda \equiv\left(2 E_{e} / m_{\pi}\right) \sin ^{2}\left(\theta_{e \gamma} / 2\right)$ for regions I and II (top panel) and of the photon energy for regions I and III (bottom panel). Solid lines: Results of GEANT3 calculations for the best-fit values of $F_{V}, F_{A}$, and $a$. 
TABLE II. Dominant sources of systematic and normalization uncertainties. They are precise determination and simulation of the trigger thresholds, energy calibration for different types of particles, radiative corrections to the acceptance, and number of the $\pi_{e 2}$ events. Remaining sources include uncertainties in the signal separation from prompt hadronic and $\pi_{\beta}$ backgrounds.

\begin{tabular}{lccc}
\hline \hline \multicolumn{1}{c}{ Source of uncertainty } & & $\begin{array}{c}\text { Region } \\
\text { II }(\%)\end{array}$ & III (\%) \\
\hline MC of trigger threshold & 0.57 & 0.37 & 1.22 \\
Calorimeter energy calibration & 0.45 & 0.38 & 0.60 \\
Corr. to $\pi_{e 2}$ acceptance & 0.20 & 0.20 & 0.20 \\
Num. of $\pi_{e 2}$ events & 0.13 & 0.13 & 0.13 \\
All other sources combined & 0.19 & 0.02 & 0.05 \\
Total & 0.79 & 0.58 & 1.38 \\
\hline \hline
\end{tabular}

simulation and the background-subtracted data in regions I, II, and III. The number $N_{\pi e 2}$ was extracted from an independent fit of the time distribution of the $\pi_{e 2}$ event candidates. Upon imposing appropriate kinematic cuts and performing the background subtraction procedure, we have reconstructed $35948 \pm 194(0.54 \%)$ events in region I, $16246 \pm 331(2.0 \%)$ events in region II, and $13263 \pm 161(1.2 \%)$ events in region III, where numbers in parentheses are fractional statistical uncertainties obtained after accidental-background subtraction. The 2004 data account for only $8 \%$ of the total for region I and about $43 \%$ in regions II and III. However, because of higher accidental-background subtraction, the 2004 events in regions II and III are statistically more significant than the 1999-2001 events. More 1999-2001 events are included here than in the analysis reported in Ref. [18] thanks to a broadening of the acceptance cuts made possible by refinements in the treatment of the positron energy deposited in the target. The acceptance cuts were adjusted so as to achieve minimal overall statistical uncertainty after background subtraction. The dominant sources of the systematic uncertainties are region dependent and are summarized in Table II. Total statistical and systematic uncertainties for each region are summed in quadrature; they are given in Table III.

In order to extract the values of $F_{V}, F_{A}$, and $a$ and calculate the values of the branching ratios, we minimized the $\chi^{2}$ sum of differences between the experimental and calculated differential branching ratios over all phase space, taking into account the form factor dependence on the $e^{+} \nu$ pair invariant mass such that $F_{V}\left(q^{2}\right)=F_{V}(0) \times$ $\left(1+a q^{2}\right), F_{A}\left(q^{2}\right)=F_{A}(0)$, and $q^{2}=1-\left(2 E_{\gamma} / m_{\pi}\right)$, following the prescription of Refs. [8-10], where $a$ is the slope parameter. The experimental branching ratios $B_{i}^{\text {expt }}$ acquire a form factor dependence through the acceptances $A_{\pi e 2 \gamma}$ which are recalculated at every iteration step of the minimization.

Both theoretical and experimental values of the branching ratios are proportional to $B_{\pi e 2}$; therefore, our $\chi^{2}$ sum
TABLE III. Best-fit $\pi_{e 2 \gamma}$ branching ratios obtained with $F_{V}=$ 0.0259 (fixed), $a=0.041$ (fixed), and $F_{A}=0.0119$ (1) (fit). Experimental uncertainties in parentheses reflect both the statistical and systematic uncertainties, given above. Theoretical uncertainties are dominated by the fit uncertainty in $F_{A}$.

\begin{tabular}{cccc}
\hline \hline Region & $B^{\text {expt }} \times 10^{8}$ & $B^{\text {theor }} \times 10^{8}$ & $B^{\text {expt }} / B^{\text {theor }}$ \\
\hline$A$ & $2.614(21)$ & $2.599(11)$ & $1.006(9)$ \\
$B$ & $14.46(22)$ & $14.45(2)$ & $1.001(15)$ \\
$C$ & $37.69(46)$ & $37.49(3)$ & $1.005(12)$ \\
$O$ & $73.86(54)$ & $74.11(3)$ & $0.997(7)$ \\
\hline \hline
\end{tabular}

assures that the values of the extracted parameters are independent of the value of $B_{\pi e 2}$.

For comparison with previous work, and to illustrate the sensitivity of our data, we performed a fit with fixed values of $F_{V}=0.0259$ [6] and $a=0.041$ [26], yielding $F_{A}=$ $(119 \pm 1) \times 10^{-4}$ for the free parameter, with $\chi_{+}^{2} /$ dof $=$ 0.96 . This result represents a 16-fold improvement in precision over the pre-PIBETA world average [27]. Table III and Fig. 1 show the excellent agreement between the measured integral and differential branching ratios and those calculated for the best-fit parameter values, reflecting the exceptionally good description of our data by CVC and standard

$V-A$ theory in all regions of phase space. The previously reported anomalous shortage of events in region $B$ [18] is thus resolved. In this work the calorimeter energy calibration (CEC) for $\gamma$-induced showers was uncoupled from that of the $e^{+}$-induced showers, resulting in a $\sim 2 \%$ difference. The clean 2004 data set revealed this discrepancy for the first time in the $1 \mathrm{HT} \pi_{\beta}$ decay event sample. Because of the limited statistical significance of such events in the 1999-2001 set, it was not feasible to evaluate a separate $\gamma$ CEC in our original analysis. Because of the extreme sensitivity of region II to the $\gamma$ energy scale, the effect was significant only in this region. More details can be found in Ref. [28] and in a forthcoming publication.

Because of the strong dominance by the $\mathrm{SD}^{+}$term, our data are most sensitive to the combination $F_{A}+F_{V}$. This sensitivity is illustrated in Fig. 2 produced by a twoparameter $\left(F_{A}, F_{V}\right)$ fit. The best-fit value of $F_{A}$ has an empirical linear dependence on the value of $F_{V}$ parametrized as

$$
F_{A}=\left(-1.0286 F_{V}+0.03853\right) \pm 0.00014 .
$$

This expression will remain applicable as the CVCpredicted value of $F_{V}$ changes thanks to future improved measurements of $\tau_{\pi 0}$, the neutral pion lifetime [29].

We also examined the question of non- $(V-A)$ contributions to the $\pi_{e 2 \gamma}$ decay by introducing tensor coupling following the prescription of Ref. [17]. The optimal fit yields a single tensor form factor $F_{T}=(-0.6 \pm 2.8) \times$ $10^{-4}$, or $-5.2 \times 10^{-4}<F_{T}<4.0 \times 10^{-4}$ with $90 \%$ confidence. Our limits on $F_{T}$ are in excellent agreement with 


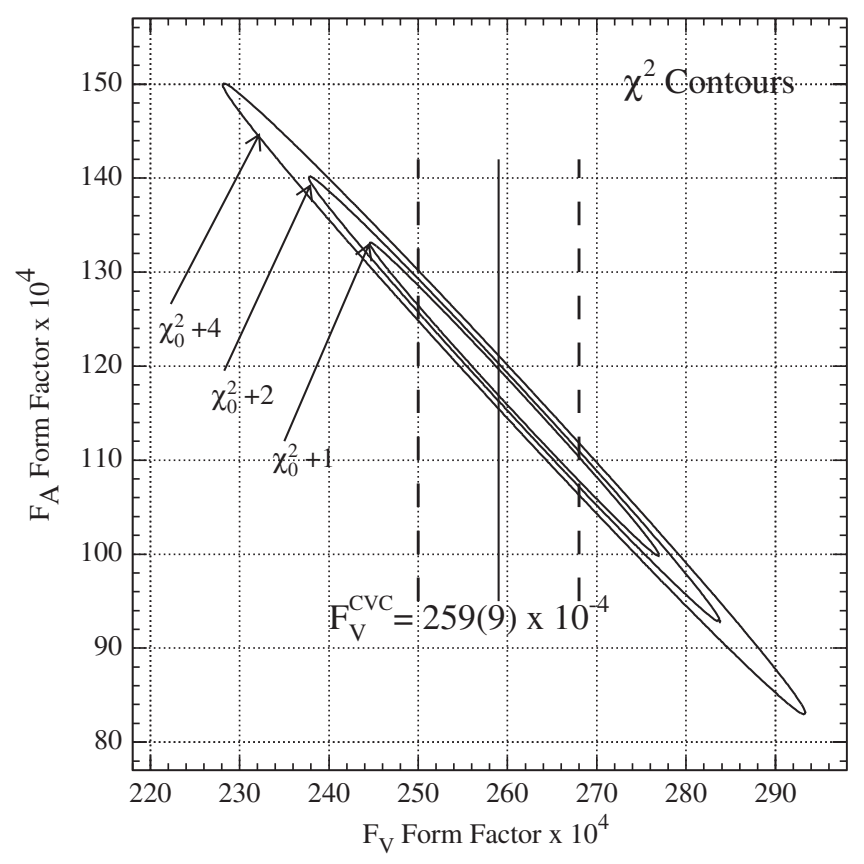

FIG. 2. Contour plot of loci of constant $\chi^{2}$ for the minimum value $\chi_{0}^{2}$ plus 1,2 , and 4 units, respectively, in the $F_{A}-F_{V}$ parameter plane, keeping the parameter $a=0.041$. The range of the CVC prediction $F_{V}=0.0259(9)$ is indicated.

Refs. [26,30] but are 2 orders of magnitude more constraining than the results reported by the ISTRA group [16,31].

Finally, in an unconstrained $V-A$ fit we released all three parameters $F_{A}, F_{V}$, and $a$ simultaneously and obtained $F_{A}=0.0117(17), \quad F_{V}=0.0258(17)$, and $a=$ $0.10(6)$. These results are nearly identical to those of the two-parameter $\left(F_{A}, F_{V}\right)$ fit presented in Fig. 2 and Eq. (2).

In summary, our results (i) are in excellent agreement with the CVC hypothesis prediction for $F_{V}$, (ii) represent a 14-fold improvement in the precision of $F_{V}$ and a similar improvement in $F_{A}$, and (iii) provide the first ever measurement of the charged pion form factor slope parameter $a$, also found to be consistent with CVC. The PEN experiment [32], currently under way at PSI, will add substantially to our $\pi_{e 2 \gamma}$ data set. Our best-fit value of $F_{A}$ agrees well with ChPT calculations, tending to the top of the reported range [7-9]. However, a more precise measurement of $\tau_{\pi 0}$ is needed in order that the sensitivity of our data, expressed in Eq. (2), be put to full use in determining $F_{A}$. We use our form factor results to evaluate the pion polarizability $\alpha_{E}$ and the ChPT parameter sum $L_{9}^{r}+L_{10}^{r}$ at leading order as follows: Using the one-parameter fit, we obtain $\alpha_{E}=-\beta_{M}=2.78(2)_{\text {expt }}(10)_{F_{V}} \times 10^{-4} \mathrm{fm}^{3}$, and $L_{9}^{r}+L_{10}^{r}=0.00145(1)_{\text {expt }}(5)_{F_{V}}$, where the first uncertainty comes from the fit and the second from the current CVC-derived value of $F_{V}$. Alternatively, we get $\alpha_{E}=$ $2.7\left({ }_{-5}^{+6}\right) \times 10^{-4} \mathrm{fm}^{3}$ and $L_{9}^{r}+L_{10}^{r}=0.0014\left({ }_{-2}^{+3}\right)$ based on our unconstrained fit of $F_{A}$ and $F_{V}$. In addition, we use the latter fit result and CVC to make an independent determination of the neutral pion lifetime: $\tau_{\pi 0}=(8.5 \pm 1.1) \times$ $10^{-17} \mathrm{~s}$.

We thank Z. Hochman for critical help in preparing and carrying out the measurements, M. V. Chizhov for valuable discussions and theoretical guidance, and J. Portoles and V. Mateu for their timely calculation of the charged pion form factor slope. The PIBETA experiment has been supported by the U.S. National Science Foundation, the U.S. Department of Energy, the Paul Scherrer Institute, and the Russian Foundation for Basic Research.

*Corresponding author. mab3ed@virginia.edu. ${ }^{\dagger}$ Corresponding author. pocanic@virginia.edu.

[1] E. Frlež et al., Nucl. Instrum. Methods Phys. Res., Sect. A 526, 300 (2004).

[2] D. A. Bryman et al., Phys. Rep. 88, 151 (1982).

[3] V. G. Vaks and B. L. Ioffe, Nuovo Cimento 10, 342 (1958).

[4] S. S. Gershtein and Ya. B. Zeldovich, Zh. Eksp. Teor. Fiz. 29, 698 (1955) [Sov. Phys. JETP 2, 576 (1956)].

[5] R. P. Feynman and M. Gell-Mann, Phys. Rev. 109, 193 (1958).

[6] C. Amsler et al., Phys. Lett. B 667, 1 (2008).

[7] B. R. Holstein, Phys. Rev. D 33, 3316 (1986).

[8] J. Bijnens and P. Talavera, Nucl. Phys. B489, 387 (1997).

[9] C. Q. Geng et al., Nucl. Phys. B684, 281 (2004).

[10] H. Pichl and R. Unterdorfer, Eur. Phys. J. C 55, 273 (2008).

[11] P. Depommier et al., Phys. Lett. 7, 285 (1963).

[12] A. Stetz et al., Nucl. Phys. B138, 285 (1978).

[13] A. Bay et al., Phys. Lett. B 174, 445 (1986).

[14] L. E. Piilonen et al., Phys. Rev. Lett. 57, 1402 (1986).

[15] C. A. Dominguez and J. Solc, Phys. Lett. B 208, 131 (1988).

[16] V. N. Bolotov et al., Phys. Lett. B 243, 308 (1990).

[17] M. V. Chizhov, Phys. Part. Nucl. Lett. 2, 193 (2005).

[18] E. Frlež et al., Phys. Rev. Lett. 93, 181804 (2004).

[19] D. Počanić et al., Phys. Rev. Lett. 93, 181803 (2004).

[20] http://pibeta.phys.virginia.edu/.

[21] Y. M. Bystritsky, E. A. Kuraev, and E. P. Velicheva, Phys. Rev. D 69, 114004 (2004).

[22] B. A. VanDevender, Ph.D. thesis, University of Virginia, 2005, p. 35.

[23] W. J. Marciano and A. Sirlin, Phys. Rev. Lett. 71, 3629 (1993).

[24] V. Ciriglano and I. Rosell, Phys. Rev. Lett. 99, 231801 (2007).

[25] R. Brun et al., GEANT 3.21, CERN, Geneva, 1994.

[26] J. Portoles and V. Mateu, Eur. Phys. J. C 52, 325 (2007).

[27] S. Eidelman et al., Phys. Lett. B 592, 1 (2004).

[28] M. Bychkov, Ph.D. thesis, University of Virginia, 2005.

[29] Primex Collaboration, http://www.jlab.org/primex/.

[30] M. B. Voloshin, Phys. Lett. B 283, 120 (1992).

[31] A. A. Poblaguev, Phys. Rev. D 68, 054020 (2003).

[32] http://pen.phys.virginia.edu/. 